\title{
Use of an electronic medical record to monitor efficacy of diabetes care in out-patients in a central hospital in Malawi: Patterns of glycaemic control and lessons learned
}

\section{Theresa J Allain ${ }^{1}$, Grieves Mang'anda ${ }^{2}$, Marrianne Kasiya ${ }^{2}$, Patricia Khomani $^{3}$, Ndaziona P Banda ${ }^{2}$, Andrew Gonani ${ }^{4}$, Ingrid Peterson ${ }^{5}$, Gavin Dreyer $^{6}$}

1.University Hospitals Bristol Teaching Trust, Bristol, UK

2.College of Medicine, University of Malawi, Private Bag 360, Chichiri, Blantyre 3, Malawi

3.Baobab Health Trust, P.O. Box 31797, Lilongwe, Malawi

4.Ministry of Health, Queen Elizabeth Hospital Blantyre, Malawi

5.Malawi Liverpool Wellcome Trust Clinical Research Programme, PO Box Blantyre, Malawi

6.Renal Unit, Barts Health NHS Trust, London, UK

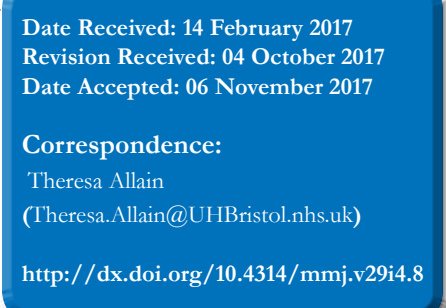

\begin{abstract}
The Malawian health sector has a strong tradition of systematic data collection for monitoring and evaluation of large-scale services. A highly successful adapted Directly Observed Treatment, Short course "DOTS" framework, based on patient registers and paperbased mastercards was introduced to facilitate the management and monitoring of the scale up of antiretroviral therapy. Subsequently, a simple, touch-screen based electronic medical record system (EMRs) was effectively introduced at high burden ART sites. Based on this model, in 2010, a diabetes specific EMRs was introduced in the diabetes clinic at Queen Elizabeth Central Hospital. In this paper we report on the first 3 years experience with the diabetes EMRs. We highlight the strengths and weaknesses of the diabetes EMRs and present data on glycaemic control recorded in the system.
\end{abstract}

\section{Background}

Low and middle-income countries (LMICs) are facing a significant rise in the number of patients with noncommunicable disease (NCD) risk factors. Rapid scaling up of services is urgently needed, but remain a challenge where resources are limited. Effective monitoring and evaluation of developing services is necessary to ensure the quality of service offered and clinical effectiveness.

The Malawian health sector has a strong tradition of systematic data collection for monitoring and evaluation of large-scale services. A highly successful adapted Directly Observed Treatment, Short course, "DOTS" framework, based on patient registers and paper-based mastercards was introduced to facilitate the management and monitoring of the scale up of antiretroviral therapy (ART) $)^{1,2}$. Within that programme a simple, touch-screen based electronic medical record system (EMRs) was effectively introduced at high burden sites ${ }^{3}$.

Prior to 2008, diabetes care in Malawi was only available at central hospitals and was basic ${ }^{4}$. A number of interventions (training of nurse specialists, introduction of protocols, and staff training) led to a rapid improvement in quality of care in the largest clinic in the country, based at Queen Elizabeth Central Hospital in Blantyre. As a model for the scale up of diabetes services nationally, a similar "DOTS" framework was introduced to that which had been successfully used for the ART scale up. Initially this was a paper-based system of registers and mastercards but with rapid expansion of the clinic an electronic medical record system (EMRs) was introduced in $2010^{5}$. This has facilitated consistent medical care at an individual level and allowed ongoing monitoring and evaluation of the service.
The aim of this study was to use data collected in the EMRs to explore clinical and operational outcomes in the clinic. The specific aims were to describe the demographic characteristics of patients attending the clinic and examine patterns of glycaemic control from the time of first attendance at the clinic. Operational outcomes included the ability to generate quarterly cohort reports and the number of patients undergoing complication screening.

\section{Methods}

\section{Study design and setting}

We conducted a retrospective, observational study of both newly diagnosed and prevalent patients with a confirmed diagnosis of diabetes mellitus (DM) seen at the Queen Elizabeth Central Hospital (QECH) diabetes clinic, Blantyre, Malawi between 1/1/2010 and 31/12/2012. The Malawi College of Medicine Research Ethics Committee granted ethical approval for the study (protocol number P.03/10/917).

\section{Structure and function of the QECH diabetes clinic}

The diabetes clinic at QECH provides free at the point of access, tertiary level clinical diabetes services for the 5.8 million residents of the Southern region of Malawi. The clinic also provides primary diabetes care for residents of Blantyre living in close proximity to the hospital. Patients in the Southern region of Malawi received care for DM in their local health centres or district hospital and were referred to $\mathrm{QECH}$ if they required specialist advice on management or complications of DM. The clinic is held twice a week, seeing 50-80 patients at each session with 4-5 clinicians (doctors and clinical officers).

Patients were seen on average 3-4 times per year but this varied 
depending on clinical need. Medications were dispensed from the central hospital pharmacy at no direct cost to the patient. Medications available were basic: glibenclamide was usually available in good supply and metformin and insulin in smaller quantities so patients were often given only a proportion of their prescribed medication. Stock outs of metformin and insulin occurred several times a year and patients were given advice about the cheapest pharmacies where these could be bought locally. Many patients could not afford to do this. A clinic visit involved nurse led group education, measurement of blood pressure, height and weight and blood sampling in the morning (after an overnight fast) for fasting blood glucose measured using Keylab (Biosed, Italy) or Mindray BS120 (Mindray, China) analyser machines in the clinical chemistry laboratory at QECH. Medical reviews typically consisted of an assessment of glycaemic and blood pressure control and overall health. Protocols required that patients underwent a foot check at every visit and screening for the complications of DM (nephropathy, retinopathy) at least once a year.

\section{Data entry systems at QECH DM clinic}

On 1/1/2010, a new electronic medical record system (EMRs) was introduced at the clinic in partnership with Baobab Health Trust (BHT) to replace the paper-based system. Clinicians working in the diabetes clinic who worked with BHT to design the system suggested the EMR fields. Briefly, a touch screen clinical workstation was used at the point of care by clinic staff to manually enter data in real time during each clinic visit. Non-variable data, for example, date of birth, physical address, gender and date of diagnosis were recorded by nurses or clerical staff at first registration for new patients. For prevalent patients, the data were transcribed from historical paper records just before the EMRs went live. The EMRs' clinical workstations were connected to a central server located at QECH using a local area network. All the registered patients' data was stored in a database on the local server. Data for this study was extracted from the database into a Microsoft Excel spreadsheet by BHT's system developers. A query was run on the extracted data to anonymise all the patients' data before the data was shared for analysis. Identification of the anonymised patients was through system-generated unique patient IDs and unique clinic reference number.

\section{Data analysis}

Data was extracted from the Diabetes Clinic EMRs to obtain a patient level dataset of all clinic visit dates and the accompanying clinical data recorded on the visit. Multiple visits were thus recorded per patient. Each visit included a measure of blood glucose level and body-mass index (BMI). For analysis of glycaemic control we calculated weeks since first visit by subtracting date of the patient's registration at the clinic from visit date and dividing by 7 . Records with missing values for blood glucose or body mass index at baseline were dropped from analysis; the EMR database enforced the entry of values for the date of the patient's registration at the clinic, date of birth, and baseline HIV status. Patients who had registered at the clinic prior to the introduction of the EMR were classified as 'previously diagnosed', while patients who registered at the clinic after the introduction of the EMR were classified as 'newly diagnosed'.

To explore whether demographic or clinical factors affected the course of glycaemic control we examined the trajectories of blood glucose levels from time since registration within patient groups. To do this, we calculated the 25th, 50th (median) and 75th percentile of blood glucose level within the patient group within 4 week groupings of time since registration. For each percentile group (25th, 50th and 75th) we plotted the trajectory of blood glucose values over time. Patient groups examined were those newly diagnosed with diabetes (incident cases) vs those registered with extant diabetes (prevalent cases); among incident cases we looked at subgroup analysis of those with a baseline BMI $>25$, 1825 and $<18$, respectively and patients with a baseline age $<45$ years versus $\geq 45$ years. Descriptive statistics were generated directly from the excel sheets using Microsoft Excel.

\section{Results}

\section{Prevalent and incident cases}

During the 3 years of study, 2,470 patients were registered in the EMRs. Of these 1,112 were prevalent cases, that is known diabetics at the time of registration on the EMRs and 636 were incident cases diagnosed during the 3 years of data collection; 186 in 2010, 249 in 2011 and 201 in 2012. Data on year of diagnosis was not recorded for 722 subjects.

\section{Demographic and clinical data at registration}

1466 (59\%) subjects were female and 1004 (41\%) were male. The median age of patients was 53 years, interquartile

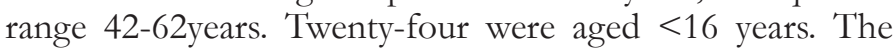
median duration of diabetes for prevalent cases was 5 years, interquartile range 2-10 years. Clinical data at registration was incomplete; for example family history was only documented for $794(32 \%)$ cases (427 positive), TB history for 1688 (68\%, 20 positive) and smoking history for 370 (7 current smokers). Data for remaining baseline clinical parameters were too incomplete to present.

\section{HIV status at the time of registration}

Data on HIV status was not documented for 1,132 (46\%) subjects. Of the remainder, 618 subjects had an unknown status, 540 had a recent non-reactive status and $180(13.5 \%$ of those for whom information was recorded) were HIV reactive. Of these $154(85.6 \%)$ were taking antiretroviral therapy (ART).

\section{Glycaemic control}

Serial glucose readings, since diagnosis, for incident cases is shown in Figure 1 (upper panel). Serial glucose measurements for prevalent cases since first recording on the EMRs is shown in figure 1 (lower panel). Newly diagnosed cases seemed to make steady progression to glucose control from 0 to 26 weeks and generally maintained $<150 \mathrm{mg} / \mathrm{dL}$ thereafter, whereas previously diagnosed subjects had overall worse control, taking longer to get to $<150 \mathrm{mg} / \mathrm{dL}$ (52 weeks), with some deterioration in control after 104 weeks.

Information on diabetes type (Type 1 or type 2), although required to be entered on the EMRs at the first visit, was largely unrecorded so subgroup analysis of newly diagnosed (incident) cases was carried out based on age and BMI. Serial glucose measurements were analysed separately in those aged $\geq 45$ years at time of diagnosis (Figure 2 upper panel) and those aged $<45$ (Figure 2 lower panel) and those with BMI $>25$ (Figure 3 upper panel) and those with BMI 18-25 (Figure 3 lower panel). Figure 2 illustrates that those aged $<45$ years start with higher blood glucose measurements but attain control by about 14 weeks. 


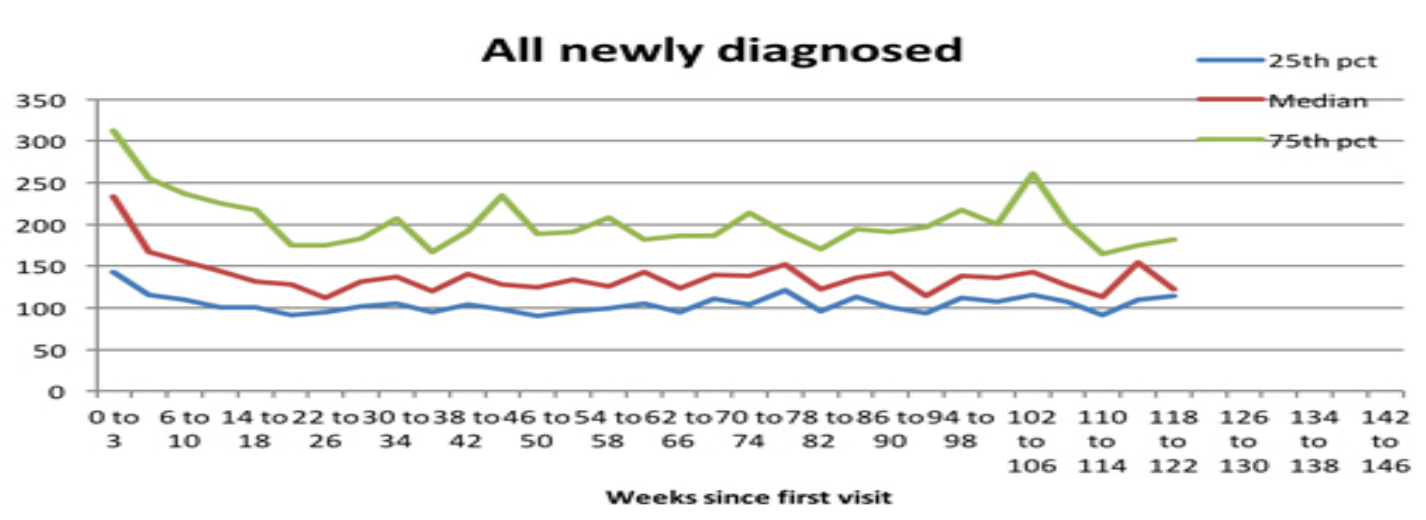

All previously diagnosed

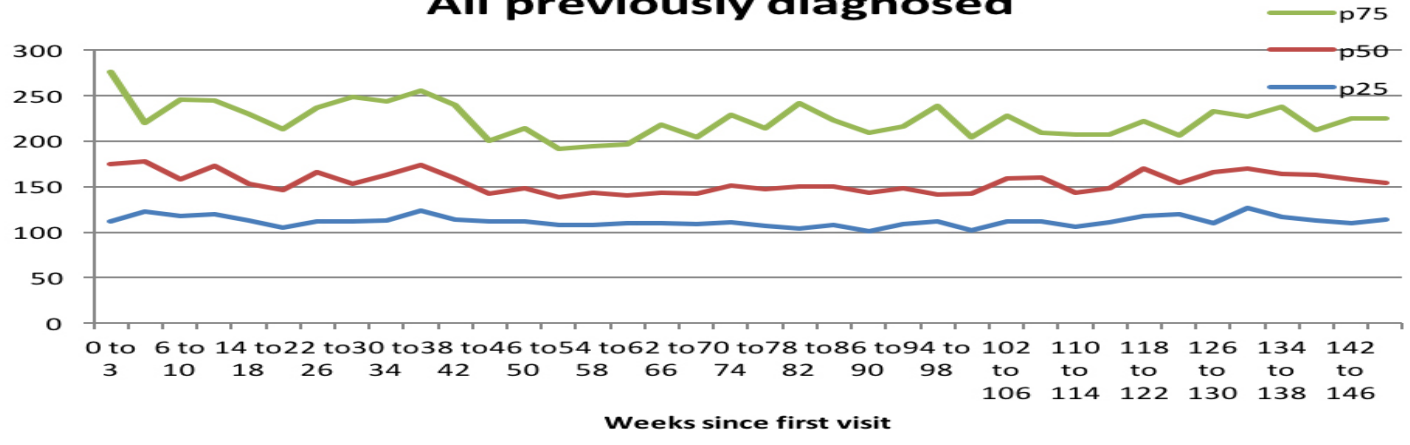

Figure 1: Serial glucose measurements for incident and prevalent cases

Age $=>45$ newly diagnosed

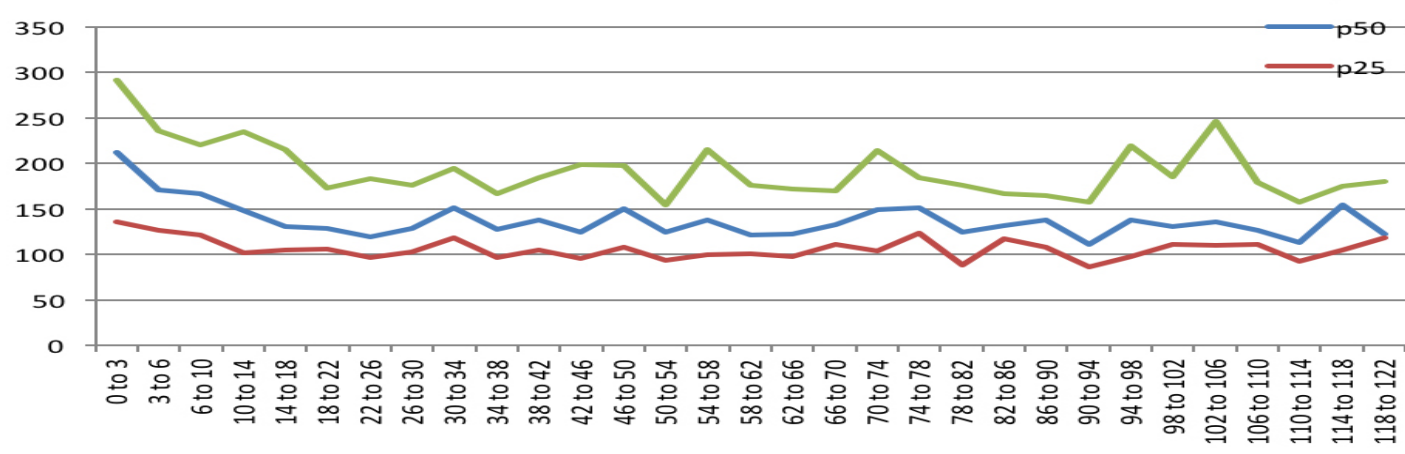

Age $<45$, newly diagnosed

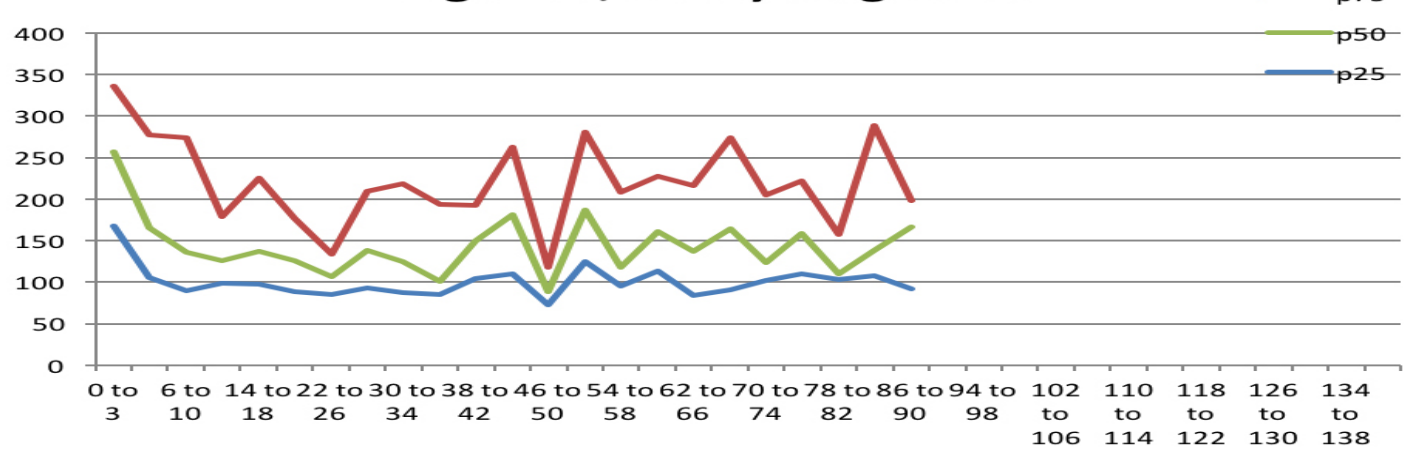

Figure 2 Serial glucose measurements stratified by age 45 years

Thereafter, from week 38 onwards glycaemic control was more variable. This group may contain a higher proportion of type 1 diabetics. Subjects aged $\geq 45$ years take longer to gain control (26 weeks), but thereafter have more consistent glycaemic levels than those aged $<45$ years. Figure 3 illustrates that those who are of normal BMI present with higher blood glucose readings and attain control by 30 weeks whereas the overweight $(\mathrm{BMI}>25)$ have lower initial glucose values, achieve glucose control by 26 weeks and maintain it over time. Again, the group with a normal BMI may include a higher proportion of type 1 diabetics. Small numbers in http://dx.doi.org/10.4314/mmj.v29i4.8 the 18-25 BMI group may account for the fluctuations in glucose levels seen later on. Data for those with BMI $<18$ is not presented as the numbers were small.

\section{Complication data}

The EMRs included fields where the complications of diabetes (retinopathy, n e p h r o p a thy, neuropathy, foot complications and macrovascular disease) could be recorded in a systematic way. The EMRs dashboard included prompts, so that a complication screen that had not been entered within the previous year was highlighted. Despite this data on the complications of DM was poorly recorded. Less than $10 \%$ of subjects had any information recorded on complications.

\section{Quarterly cohort reports}

As well as a tool to facilitate clinical management one of the main reasons for introducing the EMRs was to facilitate monitoring and evaluation, particularly through the production of quarterly cohort reports. This included data on new and cumulative registrations, outcomes (alive and on treatment, dead, transferred out, default and stop treatment), medication usage and complications. These reports were produced quarterly and forwarded to the Ministry of Health. Although registration data was more or less accurate, and useful, the other parameters were of questionable value due to missing data.

\section{Discussion}

This analysis of an EMRs with data recorded on over 2000 diabetic patients over 3 years provides some fascinating insights into the operational challenges of implementing an EMRs for a complex condition and the effectiveness of 
BMI>25, newly diagnosed

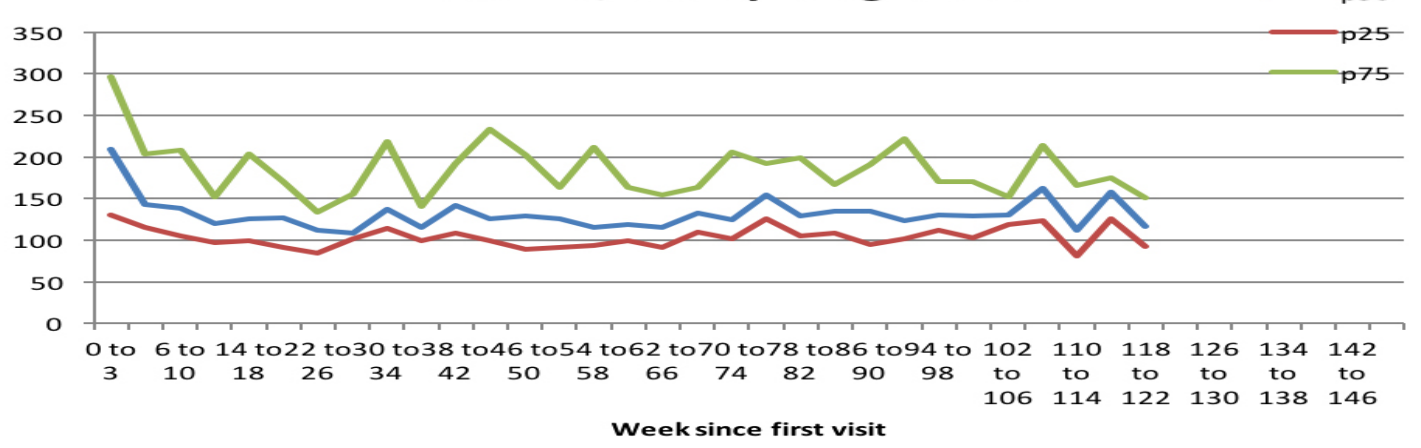

BMI 18 to 25, newly diagnosed

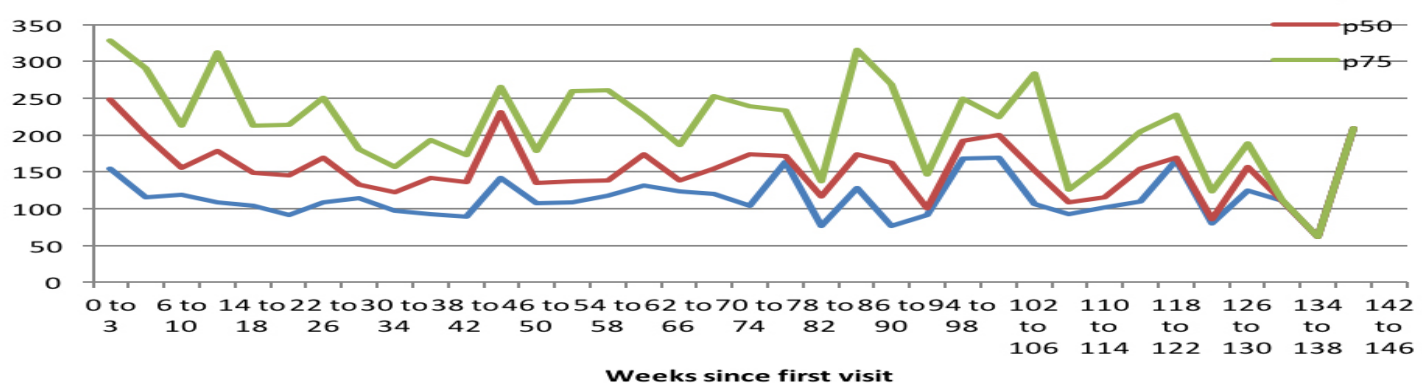

Figure 3: Serial glucose measurements stratified by BMI 18-25 and $>25$

diabetes care, in terms of glycaemic control, in a resource poor setting.

\section{Operational challenges}

Unlike the ART EMRs used for HIV care, on which the diabetes EMRs was based, where all fields are compulsory, the diabetes EMRs included only a few compulsory fields and a large number of optional fields. It has become apparent that this allows for a large amount of missing data. Furthermore, the ART EMRs is directly linked to care: thus a patient can only obtain ART through an electronic prescription and dispensing generated by the EMRs in the site where they are registered. Conversely diabetic patients can collect medication, with or without an electronic prescription, from many providers, both government and private. Thus the requirement to complete the EMRs does not link directly to outcomes. The diabetes EMRs has the functionality to print out summary stickers and prescriptions that can be stuck in the health passport (a patient kept health record that records basic information on the consultation and is used for prescribing medication. All patients in Malawi have these and bring them when attending clinics). When used frequently the stickers speed up consultation time and increase accuracy of prescribing. However, with a high turn over of clinicians who are unfamiliar with the EMRs the advantages are not recognised. The diabetes EMRs was also undoubtedly complex which was a deterrent to some users. In addition, there were often more clinicians in clinic than touch screen workstations available so some missing data was inevitable. Systematic recording of complications is a challenge in all diabetic clinics and in high volume clinics, with large numbers of patients and few clinicians; complications are not systematically screened for. This is compounded by shortages of consumable materials such as dipstick for urine protein and reagents for creatinine measurement to screen for nephropathy. The design of the diabetes EMRs, with prompts when complication screening was needed, did not have the desired effect. In this analysis we are only evaluating use of the EMRs and cannot comment on the actual clinic practices of complication screening. We know that complication rates are high $^{4,6}$ and robust processes need to be implemented to ensure that these patients are identified. However, registration data was generally well recorded and that aspect of the EMRs was one of its strengths.

\section{Glycaemic control}

The serial data on glycaemic control is unique and demonstrates the potential that EMRs can have for understanding clinical processes and clinical trajectories in patients. It is reassuring to note that most newly presenting diabetics (incident cases) achieve and maintain glycaemic control (Figure 1). The time taken (26 weeks) for acceptable control (Fasting blood glucose (FBG) less than $150 \mathrm{mg} / \mathrm{dL}$ ) to be reached reflects our clinic protocols, with an emphasis on life style changes initially for the first 3 months, in those with FBG $<300 \mathrm{mg} / \mathrm{dL}$ then gradual introduction of oral hypoglycaemic agents (OHAs) for those that are still above target. It is interesting to consider why prevalent cases seem to have worse glycaemic control. This may be a result of new patients taking on board the health messages more than our long-term patients. The education programme was stronger during the period of observation compared to before 2010. Equally, this finding may relate to the duration of DM. We know from the pathophysiology of type 2 DM that glycaemic control deteriorates over time, requiring up titration of OHAs and eventually a switch to insulin. Perhaps many of the prevalent cases needed more aggressive medical management. The observation that overall their control did improve over the first year of the EMRs suggests that the training of clinicians and introduction of treatment protocols in 2009 may have been having some impact. The deterioration in control in some subjects later on may also reflect challenges with obtaining medication since stock outs, particularly of metformin and insulin were more common in 2012.

The subgroup analyses by BMI also suggest that, in a situation where drug supplies may be constrained, lifestyle interventions have an important role to play. One of the mainstays of glycaemic control at the QECH clinic is lifestyle change, especially dietary modification and weight loss. The data suggests that it is easier to treat overweight diabetics because they will benefit most from non-pharmacological intervention. Conversely, the normal BMI patients start with higher fasting blood glucose levels and are, therefore, more likely to start on medication from their first visit, hence their glycaemic control improves more quickly. However, their subsequent control is more variable because they have less to gain by lifestyle change so treatment interruptions will 
have more impact. This group may include more varied phenotypes including type 1DM. The analysis by age reflects a similar picture.

The Ministry of Health are working with BHT to design and pilot new EMRs for non-communicable diseases namely hypertension, asthma, epilepsy, and diabetes. Based on our experience of the EMRs at QECH these new systems need to be simplified, have mainly compulsory data fields and ideally be more closely aligned to care, through registration and prescription. Clinicians who are using the EMRs need ongoing training and supervision. Continuous review of the data from the EMRs will help to identify where the gaps are coming from and can assist in identifying and working on areas where Quality Improvement Projects, within the clinic, will improve data capture.

\section{Conclusion}

In conclusion, our analysis of the first 3 years of data collected in the EMRs at Queen Elizabeth Hospital illustrates both the strengths and weaknesses of the electronic system. The potential for electronic records to facilitate clinical care, monitoring and evaluation are evident and, with better design and access to work stations for all clinicians similar EMRs could be useful for all chronic diseases. It is also apparent that clinicians using electronic systems need ongoing training, mentorship and supervision. If based on a common platform integration of electronic records for NCDs and HIV from different sites would provide a powerful database with the potential to provide a unique understanding of epidemiology, efficient service planning and improved care.

Clinical lessons from the analysis of glycaemic control suggest that different patient groups may require a different approach to management, particularly younger patients and those with lower BMIs who have more variable glycaemic control. This may require modification of protocols and alternative education messages including more intensive support to medication adherence for these groups. In those with long term diabetes protocols already reflect the need to escalate treatment as control deteriorates. These protocols need to be more closely applied and patients may benefit from modified education messages to maintain control with longer duration of diabetes.

\section{Acknowledgements}

We would like to acknowledge Shawo Mwakilama and Edith Kumwenda from Baobab Heath Trust for their support with data extraction and handling and the diabetes clinic nurses, Sheila Maliano, Madalitso Daza and Lydia Kaduya for their continuous hard work and diligence in entering baseline demographic data on the EMR.

\section{References}

1.Libamba E, Makombe S, Harries AD et al. Scaling up antiretroviral therapy in Africa: learning from tuberculosis control programmes--the case of Malawi. Int J Tuberc Lung Dis. 2005 Oct;9(10):1062-71

2.Harries AD, Nyangulu DS, Hargreaves NJ, Kaluwa O, Salaniponi FM. Preventing antiretroviral anarchy in sub-Saharan Africa. Lancet. 2001; 358(9279):410-4.

3.Douglas GP, Gadabu, OJ, Joukes S et al. Using touchscreen electronic medical record systems to support and monitor national scale-up of antiretroviral therapy in Malawi. PLoS Med. 2010; 7(8). pii: e1000319. doi: 10.1371/journal.pmed.1000319

4.Cohen DB, Allain TJ, Glover S et al. A survey of the management, control, and complications of diabetes mellitus in patients attending a diabetes clinic in Blantyre, Malawi, an area of high HIV prevalence. Am J Trop Med Hyg. 2010; 83 (3): 575-81.

5.Allain TJ, van Oosterhout JJ, Douglas GP et al. Applying lessons learnt from the 'DOTS' Tuberculosis Model to monitoring and evaluating persons with diabetes mellitus in Blantyre, Malawi. Trop Med Int Health. 2011;16 (9): 1077-84.

6.Burgess PI, Allain TJ, Garcia-Finana M, Beare NA, Msukwa G, Harding SP. High prevalence in Malawi of sight-threatening retinopathy and visual impairment caused by diabetes: identification of populationspecific targets for intervention. Diabet Med. 2014; 31(12): 1643-50. 